\title{
CHARACTERISTICS OF INDEX ONE ORBITS OF MORSE-SMALE DIFFEOMORPHISMS
}

\author{
RICHARD A. HOLMGREN
}

(Communicated by Kenneth R. Meyer)

\begin{abstract}
We use Lyapunov functions to find restrictions on the periodic data of index one orbits of Morse-Smale diffeomorphisms. The resulting structure is then used to find connections between index one orbits.
\end{abstract}

Morse-Smale diffeomorphisms are an important class of dynamical systems which can be characterized as those diffeomorphisms whose nonwandering sets consist of finitely many hyperbolic periodic points with transverse intersection. In [1], Franks presents the Morse inequalities which demonstrate a topological restriction on the periodic data of such diffeomorphisms. In this paper, we outline another restriction on the index one orbits of Morse-Smale diffeomorphisms. In addition, we demonstrate a method for finding connections between index one orbits and orbits of index zero or one. The concept of filtration tree introduced below was contained in slightly different form in [3].

\section{Preliminaries}

Let $f: M \rightarrow M$ be a Morse-Smale diffeomorphism of a compact connected manifold $M$. To each orbit $\gamma_{i}$ of $f$ we associate the triple $\left(p_{i}, u_{i}, \Delta_{i}\right)$, where $p_{i}$ is the period of $\gamma_{i}, u_{i}$ is the dimension of $W^{u}\left(\gamma_{i}\right)$, the unstable manifold of $\gamma_{i}$, and $\Delta_{i}$ is the orientation type of $\gamma_{i} . \Delta_{i}=1$ if $f^{p i}$ preserves the orientation of $W^{u}\left(\gamma_{i}\right)$ and equals -1 otherwise. The set of triples $\left\{\left(p_{i}, u_{i}, \Delta_{i}\right)\right\}$ is called the periodic data of $f$. We recall a Lyapunov function for $f$ is a continuous function $\psi: M \rightarrow \mathbf{R}$ satisfying

(1) $\psi(f(x))<\psi(x)$, when $x$ is not a periodic point, and

(2) if $x$ and $y$ are periodic points, $\psi(x)=\psi(y)$ if and only if $x$ and $y$ are in the same orbit of $f$.

In [2], there is a nice exposition of the proof of the following.

1.1. Theorem. There exists a Lyapunov function $\psi$ for $f$. Further, if $\alpha$ and $\gamma$ are orbits of $f$ and $\alpha \neq \gamma$, the only restrictions on the value of $\psi$ at $\alpha$ and $\gamma$ is $\psi(\alpha)<\psi(\gamma)$ if $W^{s}(\alpha) \cap W^{u}(\gamma) \neq \varnothing$.

Received by the editors September 25, 1989 and, in revised form, October 16, 1989. 1980 Mathematics Subject Classification (1985 Revision). Primary 58F09. 
If $\psi$ is a Lyapunov function for $f, a \in \mathbf{R}$, and $\psi^{-1}(a)$ does not include an orbit of $f$, then $N=\psi^{-1}((-\infty, a])$ is a submanifold of $M, f(N) \subset \operatorname{int}(N)$, and $\operatorname{dim}(M)=\operatorname{dim}(N)$ if $N \neq \varnothing$. If $A$ is a component of $N$ we define $W^{s}(A)=\bigcup_{x \in A^{\prime}} W^{s}(x)$ where $A^{\prime}$ is the set of periodic points contained in $A$. $W^{u}(A)$ is defined similarily.

\section{THE MAIN RESULTS}

Let $f: M \rightarrow M$ be a Morse-Smale diffeomorphism and let $\left\{\gamma_{1}, \ldots, \gamma_{k}\right\}$ be the index one orbits of $f$. We will construct a filtration of these orbits. Our results follow from an examination of the structure of this filtration.

2.1. Definition. Let $\psi: M \rightarrow \mathbf{R}$ to be a Lyapunov function which satisfies the following conditions:

(1) $\psi\left(\gamma_{i}\right)=i$.

(2) If $\alpha$ is an index zero orbit of $f$, then $\psi(\alpha)<0$.

(3) If $\lambda$ is an orbit of whose index is greater than one, then $\psi(\lambda)>k$.

Let $\varepsilon>0$ be such that the only orbit contained in $\psi^{-1}((i-2 \varepsilon, i+2 \varepsilon))$ is $\gamma_{i}$. We define the filtration $N_{0} \subset N_{1} \subset \cdots \subset N_{k}$ by $N_{i}=\psi^{-1}((-\infty, i+\varepsilon])$ for $i=0,1, \ldots, k$.

Let $N_{i}$ be an element of the filtration $N_{0} \subset N_{1} \subset \cdots \subset N_{k}$. The reader will note it is possible to choose a Lyapunov function so that some of the connected components of $N_{i}$ will not contain any periodic points of $f$. We will restrict our attention to trapping components which we define to be components of $N_{i}$ containing at least one periodic point of $f$. Note, if $A$ is a component of $N_{i}$ which is not a trapping component. then $A$ does not contain a periodic point of $f$, and so $f^{m}(A) \cap A=\varnothing$ for all $m>0$.

Let $A_{1}$ and $A_{2}$ be two trapping components of $N_{i}$. As $f^{m}\left(N_{i}\right) \subset N_{i}$ and $f^{m}\left(A_{1}\right)$ is connected, it follows that $f^{m}\left(A_{1}\right) \cap A_{2} \neq \varnothing$ implies $f^{m}\left(A_{1}\right) \subset A_{2}$. Thus it makes sense to talk about the orbit structure of the trapping components of $N_{i}$. We say $A_{1}$ and $A_{2}$ are in the same orbit if and oniy if $f^{m}\left(A_{1}\right) \subset A_{2}$ for some positive integer $m$. If $A \in \omega$, an orbit in $N_{i}$, then the period of $A$ is equal to the period of $\omega$ and is defined by

$$
\operatorname{per}(A)=\operatorname{per}(\omega)=\min \left\{m \in \mathbf{Z}^{+} \mid f^{m}\left(A_{1}\right) \subset A_{1}\right\} .
$$

2.2. Proposition. $N_{0}$ has the orbit structure of the set of periodic sinks of $f$. That is there exists a 1-1, onto correspondence between the orbits of $N_{0}$ and the orbits of the periodic sinks which commutes with $f$.

Proof. By definition, $N_{0}$ contains all the periodic sinks and no other periodic points of $f$. By definition, each trapping component of $N_{0}$ must contain at least one periodic sink. Since $N_{0}$ is contained in the union of the stable manifolds of the periodic sinks and the stable manifolds are open, it is clear that there is at most one sink in each trapping component of $N_{0}$. The result follows. 
Now let us examine the orbit structure of $N_{i}$, for $i>0$.

2.3. Definitions. Let $x \in \gamma_{i}$. As $W^{u}(x)$ is homeomorphic to $\mathbf{R}, W^{u}(x) \backslash\{x\}$ contains two components which we will denote $W_{-}^{u}(x)$ and $W_{+}^{u}(x)$. Define $A_{+}(x)$ to be the trapping component of $N_{i-1}$ satisfying $W_{+}^{u}(x) \subset W^{s}\left(A_{+}(x)\right)$. Similarily, define $A_{-}(x)$ to be the trapping component of $N_{i-1}$ satisfying $W_{-}^{u}(x) \subset W^{s}\left(A_{-}(x)\right)$. When it is possible to do so without confusion we will label $A_{+}(x)$ and $A_{-}(x), A_{+}$and $A_{-}$, respectively.

\subsection{Proposition. $A_{+}(x)$ and $A_{-}(x)$ are well defined.}

Proof. It suffices to prove the proposition for $A_{+}(x)$. Let $x \in \gamma_{i}$ and fix $y \in W_{+}^{u}(x)$. As $\psi(y)<i$, there exists $m>0$ such that $f^{m}(y) \subset N_{i-1}$. Without loss of generality we will assume that $m$ is divisible by the periods of all the trapping components of $N_{i-1}$ and by the period of $x$. Let $A$ be the trapping component of $N_{i-1}$ which contains $f^{m}(y)$. Since the period of $A$ divides $m, y \in W^{s}(A)$. Now let $\alpha$ be an arc in $W_{+}^{u}(x)$ with endpoints $y$ and $f^{m}(y)$. For sufficiently large $n, f^{m n}(\alpha)$ will be contained in $N_{i-1}$. As $\alpha$ is connected, $f^{m n}(\alpha)$ must lie entirely in one trapping component of $N_{i-1}$; namely, $A$. Hence, $\alpha \in W^{s}(A)$. As all points in $W_{+}^{u}(x)$ are contained in $f^{m n}(\alpha)$ for some integer $n$, it follows that $W_{+}^{u}(x)$ is entirely contained in $W^{s}(A)$. Clearly, $A$ is the desired trapping component $A_{+}(x)$.

2.5. Proposition. Let $x \in \gamma_{i}$. If $x$ is orientation preserving, then the periods of $A_{+}$and $A_{-}$divide the period of $x$. If $x$ is orientation reversing then $A_{+}$ and $A_{-}$are in the same orbit of $N_{i}$ and the period of $A_{+}$(and $A_{-}$) divides twice the period of $x$. Further, if $x$ is orientation reversing and the period of $A_{+}$divides the period of $x$, then $A_{+}=A_{-}$.

Proof. Suppose $\Delta(x)=1$ and $\operatorname{per}(x)=p$. Then $f^{p}\left(W_{+}^{u}(x)\right)=W_{+}^{u}(x)$. Hence, $f^{p}\left(A_{+}\right) \cap A_{+} \neq \varnothing$ which implies $f^{p}\left(A_{+}\right) \subset A_{+}$. Similarily, we see that $f^{p}\left(A_{-}\right) \subset A_{-}$. So, $\operatorname{Per}\left(A_{+}\right)$and $\operatorname{Per}\left(A_{-}\right)$both divide $p=\operatorname{per}(x)$.

Now suppose $\Delta(x)=-1$ and $\operatorname{per}(x)=p$. Since $f^{p}\left(W_{+}^{u}(x)\right)=W_{-}^{u}(x)$, $f^{p}\left(A_{+}\right) \cap A_{-} \neq \varnothing$. Thus, $f^{p}\left(A_{+}\right) \subset A_{-}$and $A_{+}$and $A_{-}$are in the same orbit of $N_{i-1}$. Now $f^{2 p}\left(W_{+}^{u}(x)\right)=W_{+}^{u}(x)$ so $f^{2 p}\left(A_{+}\right) \cap A_{+} \neq \varnothing$ which implies $f^{2 p}\left(A_{+}\right) \subset A_{+}$. Therefore, $\operatorname{per}\left(A_{+}\right)$divides $2 p$. Note, if $\operatorname{per}\left(A_{+}\right)$divides $p$, then $f^{p}\left(A_{+}\right) \subset A_{-}$implies $A_{+}=A_{-}$.

Now the orbit structure of $N_{i}$ can be determined from the orbit structure of $N_{i-1}$ and $\gamma_{i}$ via the following theorem.

2.6. Theorem. Let $\left\{\omega_{1}, \omega_{2}, \ldots \omega_{m}\right\}$ be the set of orbits of trapping components of $N_{i-1}$, let $x \in \gamma_{i}$, and let $p=\operatorname{per}(x)$.

(i) Suppose $\Delta(x)=1$, and $A_{-}$and $A_{+}$are in separate orbits of $N_{i-1}$, say $\omega_{1}$ and $\omega_{2}$, then $N_{i}$ has orbits $\left\{\omega, \omega_{3}^{\prime}, \omega_{4}^{\prime}, \ldots, \omega_{m}^{\prime}\right\}$ with periods so 
that

$$
\begin{aligned}
& \operatorname{per}\left(\omega_{i}^{\prime}\right)=\operatorname{per}\left(\omega_{i}\right) \text { for } i=3,4, \ldots, m, \\
& \operatorname{per}(\omega)=\operatorname{gcd}\left[\operatorname{per}\left(\omega_{1}\right), \operatorname{per}\left(\omega_{2}\right)\right] .
\end{aligned}
$$

(ii) Suppose $\Delta(x)=1$, and $A_{-}$and $A_{+}$are in the same orbit of $N_{i-1}$, say $\omega_{1}$, then $N_{i}$ has orbits $\left\{\omega, \omega_{2}^{\prime}, \ldots \omega_{m}^{\prime}\right\}$ with periods so that

$$
\begin{aligned}
& \operatorname{per}\left(\omega_{i}^{\prime}\right)=\operatorname{per}\left(\omega_{i}\right) \quad \text { for } i=2,3, \ldots, m, \\
& \text { and } \operatorname{per}(\omega) \text { divides } \operatorname{per}\left(\omega_{1}\right) .
\end{aligned}
$$

(iii) Suppose $\Delta(x)=-1$, and $\operatorname{per}\left(A_{+}\right)$divides $2 p$ but does not divide $p$, then $N_{i}$ has orbits $\left\{\omega, \omega_{2}^{\prime}, \ldots \omega_{m}^{\prime}\right\}$ with periods so that

$$
\begin{aligned}
& \operatorname{per}\left(\omega_{i}^{\prime}\right)=\operatorname{per}\left(\omega_{i}\right) \quad \text { for } i=2,3, \ldots, m, \\
& \text { and } \operatorname{per}(\omega)=\frac{1}{2} \operatorname{per}\left(\omega_{1}\right) \text {. }
\end{aligned}
$$

(iv) Suppose $\Delta(x)=-1$, and $\operatorname{per}\left(A_{+}\right)$divides $p$, then $N_{i}$ has orbits $\left\{\omega_{1}^{\prime}\right.$, $\left.\omega_{2}^{\prime}, \ldots \omega_{m}^{\prime}\right\}$ with periods so that

$$
\operatorname{per}\left(\omega_{i}^{\prime}\right)=\operatorname{per}\left(\omega_{i}\right) \quad \text { for } i=1,2,3, \ldots, m \text {. }
$$

In the proof of the theorem we will use:

2.7. Lemma. Let $x \in \gamma_{i}$ and define $V_{i}=N_{i-1} \cup\left[\cup_{a} W^{u}\left(f^{a}(x)\right)\right]$. Then the orbit structure of $N_{i}$ is the same as the orbit structure of $V_{i}$. That is, there exists a 1-1, onto correspondence between the orbits of $V_{i}$ and the orbits of $N_{i}$ which commutes with $f$.

Proof of the lemma. If $V_{i}$ has only one trapping component we are done as this implies $N_{i}$ has only one trapping component. If not, let $3 \delta$ be the minimum distance between any two disjoint trapping components of $V_{i}$. As $V_{i}$ is closed and compact, $3 \delta>0$. Let $V_{i}(\delta)$ be a $\delta$ neighborhood of $V_{i}$ and let $\hat{V}_{i}=$ $N_{i} \cap V_{i}(\delta)$. Clearly $\hat{V}_{i}$ and $V_{i}$ have the same orbit structure. Also, $f^{k}\left(N_{i}\right)$ and $N_{i}$ have the same orbit structure for all integers $k$. As $\hat{V}_{i} \subset N_{i}$, to complete the proof it suffices to show $f^{k}\left(N_{i}\right) \subset \hat{V}_{i}$ for some $k$.

Let $z \in N_{i}$. Since all points in $M$ belong to the stable manifold of some periodic point and $\psi(z)<i, z$ is contained in the stable manifold of some periodic point contained in $V_{i}$. Hence, there exists an integer $k^{\prime}$ such that $f^{k^{\prime}}(z) \in \hat{V}_{i}$. As $N_{i}$ is compact this implies there exists an integer $k$ such that $f^{k}\left(N_{i}\right) \subset \hat{V}_{i}$.

Proof of the theorem. Let $x \in \gamma_{i}$, and let $p=\operatorname{per}(x)$. By the preceding lemma it suffices to prove the theorem for

$$
V_{i}=N_{i-1} \cup\left[\bigcup_{a} W^{u}\left(f^{a}(x)\right)\right] \text {. }
$$


(i) Suppose $\Delta(x)=1$, that is $x$ is orientation preserving, and $A_{-}$and $A_{+}$are in different orbits of $N_{i-1}$, say $A_{+} \in \omega_{1}$ and $A_{-} \in \omega_{2}$. In $V_{i}=$ $N_{i-1} \cup\left[\bigcup_{a} W^{u}\left(f^{a}(x)\right)\right] A_{+}$and $A_{-}$are connected by $W^{u}(x)$. Let $\omega=$ $\cup_{a \geq 0}\left[f^{a}\left(A_{+} \cup W^{u}(x) \cup A_{-}\right)\right]$. To complete the proof of (i) it suffices to show $\omega$ has $d$ trapping components which are cyclically permuted by $f$, where $d=$ $\operatorname{gcd}\left[\operatorname{per}\left(A_{+}\right), \operatorname{per}\left(A_{-}\right)\right]$.

Let $p_{+}=\operatorname{per}\left(A_{+}\right)$and $p_{-}=\operatorname{per}\left(A_{-}\right)$. Note $A_{+}$is connected to $f^{a}\left(A_{-}\right)$by $f^{b p_{+}}\left(W^{u}((x))\right.$ if and only if $a \equiv b p_{+} \bmod \left(p_{-}\right)$This follows from $f^{b p_{+}}\left(W_{+}^{u}(x)\right)$ $\subset W^{s}\left(f^{b p_{+}}\left(A_{+}\right)\right)=W^{s}\left(A_{+}\right), f^{b p_{+}}\left(W_{-}^{u}(x)\right) \subset W^{s}\left(f^{b p_{+}}\left(A_{-}\right)\right)$, and $W^{s}\left(f^{b p_{+}}\left(A_{-}\right)\right)$ $\subset W^{s}\left(f^{a}\left(A_{-}\right)\right.$if and only if $a \equiv \bmod \left(p_{+}\right)$. So, if $d=\operatorname{gcd}\left(p_{+}, p_{-}\right), A_{+}$is connected to $f^{a}\left(A_{-}\right)$by an iterate of $W^{u}(x)$ if and only if $a=b d$ for some integer $b$. Similarily, $A_{-}$is connected to $f^{a}\left(A_{-}\right)$by an iterate of $W^{u}(x)$ if and only if $a=b d$ for some integer $b$.

Now let $j \in\{1, w, \ldots, d-1\}$ where $d=\operatorname{gcd}\left(p_{+}, p_{-}\right)$. By an argument similar to the one used above we can show $f^{j}\left(A_{+}\right)$is connected to $f^{a+j}\left(A_{-}\right)$ by an iterate of $W^{u}(x)$ if and only if $a=b d$ for some integer $b$. Our claim for the set $\omega$ and hence the proof of (i) follows.

(ii) Suppose $\Delta(x)=1$ and that $A_{+}$and $A_{-}$are in the same orbit of $N_{i-1}$, say $\omega_{1}$. Let $\left\{A_{1}, A_{2}, \ldots, A_{n}\right\}$ be the trapping components of $\omega_{1}$ indexed such that $f\left(A_{i}\right)=A_{i+1}$. Recall, $n$ is divisible by $p$. Without loss of generality we will assume $A_{+}=A_{1}$. Consider the orbit, $\omega$, of $V_{i}=N_{i-1} \cup\left[\bigcup_{a} W^{u}\left(f^{a}(x)\right)\right]$ defined by $\omega=\left(\bigcup_{i} A_{i}\right) \cup\left(\bigcup_{a} W^{u}\left(f^{a}(x)\right)\right)$. Clearly, if $A_{-}=A_{1}\left(=A_{+}\right)$, then $\operatorname{per}(\omega)=\operatorname{per}\left(\omega_{1}\right)$. An argument similar to the one used in the proof of (i) will show, if $A_{-}=A_{i}$ where $i \neq 1$, then $\operatorname{per}(\omega)=\operatorname{gcd}(p, i-1)$ and we are done with the proof of (ii).

(iii) Suppose $\Delta(x)=-1, \operatorname{per}\left(A_{+}\right)=p_{+}$, and $p_{+}$divides $2 p$ but does not divide $p$. That is, $p=\left(\frac{1}{2}+a\right) p_{+}$for some integer $a$. By Proposition 2.5 we know that $A_{+}$and $A_{-}$belong to the same orbit of $N_{i-1}$, say $\omega_{1}$. Then, $f^{p}\left(W_{+}^{u}(x)\right)=W_{-}^{s}(x)$ implies $f^{p}\left(A_{+}\right) \cap A_{-} \neq \varnothing$ or $f^{(1 / 2+a) p_{+}}\left(A_{+}\right) \cap A_{-} \neq \varnothing$ which implies $f^{1 / 2 p_{+}}\left(A_{+}\right) \cap A_{-} \neq \varnothing$. Hence, $f^{1 / 2 p_{+}}\left(A_{+}\right) \subset A_{-}$. By assumption there are an even number of trapping components in $\omega_{1}$. Thus, the trapping components of $\omega_{1},\left\{A_{1}, A_{2}, \ldots, A_{n}, A_{1}^{\prime}, A_{2}^{\prime}, \ldots, A_{n}^{\prime}\right\}$, can be indexed such that $A_{1}=A_{+}$and $A_{1}^{\prime}=A_{-} ; f\left(A_{i}\right)=A_{i+1}$ for $i=1,2, \ldots, n-1 ; f\left(A_{n}\right)=$ $A_{1}^{\prime} ; f\left(A i^{\prime}\right)=A_{i+1}^{\prime}$ for $i=1,2, \ldots, n-1$; and $f\left(A_{n}^{\prime}\right)=A_{1}$. Now consider the orbit, $\omega$, of $V_{i}$ defined by $\omega=\left(\bigcup_{i} A_{i}\right) \cup\left(\bigcup_{a} W^{u}\left(f^{a}(x)\right)\right) \cup\left(\bigcup_{i} A_{i}^{\prime}\right)$. It is clear that $A_{+}$is connected to $A_{-}$by $W^{u}(x)$ and, in general, $A_{i}$ is connected to $A_{i}^{\prime}$ is connected to $A_{i}^{\prime}$ by $W^{u}\left(f^{i}(x)\right)$. Hence, $\omega$ has the desired orbit structure.

(iv) Suppose $\Delta(x)=-1, \operatorname{per}\left(A_{+}\right)=p_{+}$, and $p_{+}$divides $p$. That is, $p=$ $a p_{+}$for some integer $a$. By Proposition 2.5 we know that $A_{+}=A_{-}$. So $\bigcup_{a}\left[W^{u}\left(f^{a}(x)\right)\right]$ does not connect any trapping components of $N_{i-1}$ in $V_{i}$ and the result follows. 
2.8. Proposition. $N_{k}$ contains a single trapping component. (Recall, $\gamma_{k}$ is the index one orbit with the highest Lyapunov value.)

Proof. It suffices to show: If $\varphi$ is a Luapunov function, $N=\varphi^{-1}((-\infty, a])$, and $N^{\prime}=\varphi^{-1}((-\infty, b])$, are such that $N^{\prime} \subset N, \varphi^{-1}(b)$ does not contain any periodic points, and the only orbit contained in $N \backslash N^{\prime}$ is $\gamma$ where $\gamma$ has index $\geq 2$, then $N$ is not connected if $N^{\prime}$ is not connected. From this it follows that $M$ is not connected if $N_{k}$ is not connected.

To prove the assertion let $x \in \gamma, m$ be the index of $x, p=\operatorname{per}(x)$, and $D \subset W^{u}(x)$ be an $m$-disk centered at $x$. Define $B=f^{p}(D) \backslash D$. Note, $B$ is connected as $m \geq 2$ and $f^{p}$ is an expanding map. Now there exists $n \in \mathbf{Z}$ such that $f^{n p}(B) \subset N^{\prime}$. Since $B$ is connected, $f^{n p}(B)$ is contained entirely in one trapping component of $N^{\prime}$, say $A$. This implies $W^{s}(A) \supset\left(W^{u}(x) \backslash\{x\}\right)$. Hence, none of the trapping components of $N^{\prime}$ will be connected by $W^{u}(x)$ in $N$. Thus, $N$ will have as many trapping components as $N^{\prime}$ does.

As a bookkeeping device we introduce the filtration tree. The idea is to construct a directed tree in which each node corresponds to an index one or index zero orbit. Each branch of the tree will correspond to an orbit of trapping components in some element of the filtration $N_{0} \subset N_{1} \subset \cdots \subset N_{m}$.

2.9. Definition. We define a value directed tree to be a connected acyclic graph with directed edges such that each node (vertex) has an associated integer value. The value of a node $n^{\prime}$ is denoted by $\mathscr{V}\left(n^{\prime}\right)$. A node with no edges leaving it is called a lower terminal node. A node with no edges entering it is called an upper terminal node. The remaining nodes are called intermediary nodes. We say node $n_{0}$ connects to node $n_{1}$ if there is an edge from $n_{0}$ to $n_{1}$ and we write $n_{0} \rightarrow n_{1}$. If there is a sequence of connected nodes from $n_{1}$ to $n_{t}$, $n_{1} \rightarrow n_{2} \cdots n_{t-1} \rightarrow n_{t}$, we say $n_{t}$ lies below $n_{1}$, and $n_{1}$ lies above $n_{t}$.

2.10. Definition. Let $N_{0} \subset N_{1} \subset \cdots \subset N_{m}$ be a filtration for the diffeomorphism $f$ and Lyapunov function $\psi$ as defined in 2.1. Let $\left\{\gamma_{1}, \gamma_{2}, \ldots, \gamma_{k}\right\}$ be the index one points of $f$ and let $\left\{\alpha_{1}, \alpha_{2}, \ldots, \alpha_{m}\right\}$ be the index zero points of $f$. A filtration tree, $T$, for $N_{i}$ is a value directed tree satisfying the following conditions:

(1) There is a one-to-one correspondence between the lower terminal nodes of $T$ and the index zero orbits of $f$. The value of the lower terminal node corresponding to the index zero orbit $\alpha_{i}$ is the period of $\alpha_{i}$.

(2) There is a one-to-one correspondence between the upper terminal and intermediary nodes of $T$ and the index one orbits of $f$. The value of the node corresponding to the index one orbit $\gamma_{i}$ is the period of the orbit of trapping components in $N_{i}$ containing $\gamma_{i}$.

(3) If $n_{0}$ lies below $n_{1}$ and $\gamma_{i}$ is the index one orbit corresponding to $n_{1}$, then the orbit corresponding to $n_{0}$ is contained in the orbit of trapping components in $N_{i}$ which contains $\gamma_{i}$. 
2.11. Notes. The filtration tree is dependent on the choice of Lyapunov function $\psi$. We shall say the orbit $\alpha$ lies below the orbit $\gamma$ in the filtration tree $T$ if the corresponding nodes of $T$ are so ordered.

The following proposition outlines some of the characteristics of filtration trees.

2.12. Proposition. Let $T$ be a filtration tree for $f$, then

(1) $T$ has a unique upper terminal node with value one.

(2) If $\gamma$ is the index one orbit corresponding to the node $n(\gamma)$ and $\Delta(\gamma)=1$, then there is either one or two edges leaving $n(\gamma)$ and the period of $\gamma$ is divisible by the value of the nodes to which $n(\gamma)$ connects. If there are two edges leaving $n(\gamma)$, then $\mathscr{V}(n(\gamma))=\operatorname{gcd}\left(\mathscr{V}\left(n_{1}\right), \mathscr{V}\left(n_{2}\right)\right)$. where $n_{1}$ and $n_{2}$ are the nodes to which $n(\gamma)$ connects. If there is one edge leaving, $\mathscr{V}(n(\gamma))$ divides $\mathscr{V}\left(n_{1}\right)$ where $n_{1}$ is the node to which $n(\gamma)$ connects.

(3) If $\gamma$ is the index one orbit corresponding to the node $n(\gamma)$ and $\Delta(\gamma)=$ -1 , then there is a unique edge leaving $n(\gamma)$. If $n_{1}$ is the node to which $n(\gamma)$ connects, then $\mathscr{V}\left(n_{1}\right)$ divides twice the period of $\gamma$ and $\mathscr{V}(n(\gamma))=\mathscr{V}\left(n_{1}\right)$ if $\mathscr{V}\left(n_{1}\right)$ divides the period of $\gamma$, and equals $\frac{1}{2} \mathscr{V}\left(n_{1}\right)$ otherwise.

Proof. (1) follows from the fact that $T$ is connected and Proposition 2.8. (2) and (3) follow from Theorem 2.6.

2.13. Definition. Let $\mathbf{P}$ be the periodic data of $f$. We say $\mathbf{P}$ admits the value directed tree $T$ as a filtration tree if there is a one-to-one correspondence between the index zero orbits of $f$ and the lower terminal nodes of $T$ such that the value of these nodes equals the period of the associated orbit and if there is a one-to-one correspondence between the remaining nodes of $T$ and the index one orbits of $f$ which satisfies the conditions of Proposition 2.12. Such a value directed tree is called an admissible filtration tree for $\mathbf{P}$

2.14. Example. Suppose the periodic data of the index zero and one points of $f$ is $\{(2,0,1),(4,0,1),(8,1,1),(2,1,1),(1,1,-1)\}$. Then two of the admissible filtration trees of $f$ are:
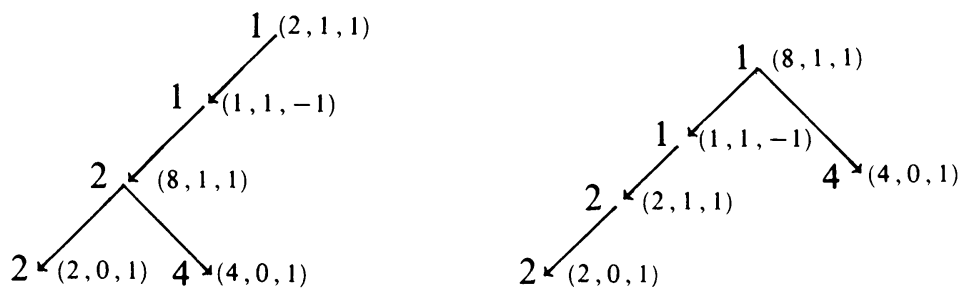

The value of the node is written to the left of the node and the periodic data of the associated orbit is written to the right of the node.

The following theorem is an immediate corollary of Proposition 2.2, Theorem 2.6, and Proposition 2.8. 
2.15. Theorem. If $\mathbf{P}$ is the periodic data of a Morse-Smale diffeomorphism, then $\mathbf{P}$ admits a filtration tree.

The filtration $N_{i}$ also yields inforination about the connections between the orbits of a Morse-Smale diffeomorphism. We say there is a connection from the orbit $\gamma$ to the orbit $\alpha$ of $f$ if $W^{s}(\alpha) \cap W^{u}(\gamma) \neq \varnothing$. Clearly, if an index zero or one orbit $\alpha$ of $f$ does not lie below the index one orbit $\gamma$ of $f$ in the filtration tree then there is no connection from $\gamma$ to $\alpha$. On the other hand, if $\alpha$ lies below $\gamma$ in the filtration tree this does not necessarily imply there is a connection. However, we can show:

2.16. Theorem. Let $\gamma$ be an index one orbit of the Morse-Smale diffeomorphism $f$. Let $\psi$ be a Lyapunov function which satisfies Definition 2.1. Denote the filtration tree determined by $\psi, T$, and denote the node of $T$ corresponding to $\gamma, n(\gamma)$. Assume $n(\gamma) \rightarrow n(\alpha)$ in $T$ where $n(\alpha)$ is the node of $T$ corresponding to the orbit $\alpha$. If $\Delta(\gamma)=1$ and $n(\alpha)$ is the only node below $n(\gamma)$ whose value divides the period of $\gamma$, or if, $\Delta(\gamma)=-1$ and $n(\alpha)$ is the only node below $n(\gamma)$ whose value divides twice the period of $\gamma$, then there is a connection from $\gamma$ to $\alpha$. That is, $W^{s}(\alpha) \cap W^{u}(\gamma) \neq \varnothing$.

Proof. Let $x \in \gamma$. If $\alpha$ is an index zero orbit, $\alpha$ is the only orbit below $\gamma$ on that branch of $T$. As the unstable manifold of an index one orbit must lie in the stable manifold of the union of the orbits which lie below it in $T$, either $W_{+}^{u}(x) \subset W^{s}(\alpha)$ or $W_{-}^{u}(x) \subset W^{s}(\alpha)$ as desired.

Now assume $\alpha$ is an index one orbit. Suppose $\Delta(\gamma)=1$ and $n(\alpha)$ is the only node below $\gamma$ in $T$ whose value divides the period of $\gamma$. Let $N^{\prime}$ be the element of the filtration. $N_{0} \subset N_{1} \subset \cdots \subset N_{k}$, associated with $\alpha$. That is, $N^{\prime}$ is the first element of the filtration which contains $\alpha$. Let $N^{\prime \prime}$ be the element of the filtration immediately below $N^{\prime}$ and let $\omega$ be the orbit of $N^{\prime}$ which contains $\alpha$.

Now choose $x \in \gamma$. By our previous work we know that either $W_{+}^{u}(x) \subset$ $W^{s}(\omega)$ or $W_{-}^{u}(x) \subset W^{s}(\omega)$. Without loss of generality we will assume $W_{+}^{u}(x)$ $\subset W^{s}(\omega)$. Also by our previous work we know $W^{u}(\alpha)$ is connecting one or two orbits of $N^{\prime \prime}$. We will assume $W^{u}(\alpha)$ connects the orbits $\omega_{1}$ and $\omega_{2}$ of $N^{\prime \prime}$ where $\omega_{2}$ may be empty. By hypothesis, the periods of $\omega_{1}$ and $\omega_{2}$ do not divide the period of $\gamma$. Note the set $W^{s}(\omega) \backslash W^{s}(\alpha)=W^{s}\left(\omega_{1}\right) \cup W^{s}\left(\omega_{2}\right)$ is open and disconnected. Hence, if $W_{+}^{u}(x) \subset W^{s}\left(\omega_{1}\right) \cup W^{s}\left(\omega_{2}\right)$ it must be contained entirely in one trapping component of $W^{s}\left(\omega_{1}\right) \cup W^{s}\left(\omega_{2}\right)$. But this is not possible as the periods of $\omega_{1}$ and $\omega_{2}$ do not divide the period of $\gamma$. Thus, $W_{+}^{u}(x) \subset W^{s}(\omega)$ implies $W^{s}(\alpha) \cap W^{u}(\gamma) \neq \varnothing$.

The proof for the case $\Delta(\gamma)=-1$ is similar.

2.17. Note. The connection of orbits is transitive. That is, if $\alpha, \gamma$, and $\lambda$ are orbits of $f$ such that there are connections from $\lambda$ to $\gamma$ and from $\gamma$ to $\alpha$, then there is a connection from $\lambda$ to $\alpha$.

Together the transitivity of connections and Theorem 2.16 allows us to find many connections if we can determine an appropriate Lyapunov function and 
filtration for the given Morse-Smale diffeomorphism. In those cases where we have only the periodic data we need to look at all admissible filtration trees.

2.18. Theorem. Let $\mathbf{P}$ be the periodic data of the Morse-Smale diffeomrophism $f$. If $\alpha$ is an index zero or one orbit which lies below the index one orbit $\gamma$ in every admissible filtration tree for $\mathbf{P}$, then there is a connection from $\gamma$ to $\alpha$. That is, $W^{s}(\alpha) \cap W^{u}(\gamma) \neq \varnothing$.

Proof. Let $\alpha$ and $\gamma$ be index one orbits of $f$ and suppose $W^{s}(\alpha) \cap W^{u}(\gamma)=\varnothing$. That is, there is no connection from $\gamma$ to $\alpha$. Then by Theorem 1.1 there exists a Lyapunov function $\varphi$ such that $\varphi(\alpha)>\varphi(\gamma)$. Further, as both $\alpha$ and $\gamma$ are index one orbits, we can assume $\varphi$ satisfies the conditions of Definition 2.1. Examination of the filtration tree $T(\varphi)$ associated with $\varphi$ will show that $\alpha$ is not below $\gamma$ in $T(\varphi)$. Hence, if $\alpha$ lies below $\gamma$ in every admissible filtration tree of $\mathbf{P}, W^{s}(\alpha) \cap W^{u}(\gamma) \neq \varnothing$.

If $\alpha$ is an index zero orbit of $f$, we will use an argument similar to that used above. However, it will be necessary to develop an alternative filtration and associated tree.

Let $\left\{\gamma_{1}, \gamma_{2}, \ldots, \gamma_{k}\right\}$ be the index one orbits of $f$ and define $\hat{\varphi}: M \rightarrow \mathbf{R}$ to be a Lyapunov function which satisfies the following conditions:

(1) $\hat{\varphi}\left(\gamma_{i}\right)=i$

(2) If $\alpha$ is an index zero orbit of $f$, then $\hat{\varphi}(\alpha)<\hat{\varphi}\left(\gamma_{i}\right)$ if and only if $W^{s}(\alpha) \cap W^{u}\left(\gamma_{i}\right) \neq \varnothing$.

(3) If $\lambda$ is an orbit whose index is greater than one, then $\hat{\varphi}(\lambda)>k$.

Let $\varepsilon>0$ be such that the only orbit contained in $\psi^{-1}((i-2 \varepsilon, i+2 \varepsilon))$ is $\gamma_{i}$. We can then define a filtration $\hat{N}_{0} \subset \hat{N}_{1} \subset \cdots \subset \hat{N}_{k}$ by $\hat{N}_{i}=\hat{\varphi}^{-1}((-\infty, i+\varepsilon])$, for $i=1,2, \ldots, k$, and $\hat{N}_{0}=\hat{\varphi}^{-1}((-\infty, 1-\varepsilon])$.

Now let $\varphi: M \rightarrow \mathbf{R}$ be a Lyapunov function which satisfies Definition 2.1 and let $N_{0} \subset N_{1} \subset \cdots \subset N_{k}$ be the filtration defined by $\varphi$. Notice $\varphi\left(\gamma_{i}\right)=\hat{\varphi}\left(\gamma_{i}\right)$. It is easy to check that the orbit structure of $\hat{N}_{i}$ is the same as the orbit structure of $N_{i} \backslash A_{i}$ where $A_{i}$ is the set of index zero orbits, $\alpha$ satisfying $\varphi(\alpha)>i$. Further, it is clear that the filtration $\hat{N}_{i}$ defines a filtration tree $\hat{T}$, which is identical to the filtration tree defined by $N_{i}$.

Now suppose $\alpha$ is an index zero orbit of $f, \gamma$ is an index one orbit of $f$, and $W^{s}(\alpha) \cap W^{u}(\gamma)=\varnothing$. That is, there is no connection from $\gamma$ to $\alpha$. Then $\alpha$ does not lie below $\gamma$ in the filtration tree $\hat{T}$ defined above. Hence, if $\alpha$ lies below $\gamma$ in every admissible filtration tree of $\mathbf{P}, W^{s}(\alpha) \cap W^{u}(\gamma) \neq \varnothing$.

\section{REFERENCES}

1. J. Franks, Homology and dynamical systems, CBMS Regional Conf. Ser. in Math., vol. 49, Amer. Math. Soc., Providence, RI, 1982.

2. _ A variation of the Poincare-Birkhoff theorem, preprint.

3. R. Holmgren, Morse-Smale diffeomorphisms of the three sphere, doctoral dissertation, Northwestern University, 1988.

Department of Mathematics, Allegheny College, Meadville, Pennsylvania 16335 